\title{
DESIGN OF SAMPLING SET FOR BANDLIMITED GRAPH SIGNAL ESTIMATION
}

\author{
Xuan Xie, Hui Feng, Junlian Jia, Bo Hu \\ Research Center of Smart Networks and Systems, Fudan University, Shanghai 200433, China \\ Emails: $\{$ xxie15, hfeng, jljia10, bohu $\} @$ fudan.edu.cn
}

\begin{abstract}
It is of particular interest to reconstruct or estimate bandlimited graph signals, which are smoothly varying signals defined over graphs, from partial noisy measurements. However, choosing an optimal subset of nodes to sample is NP-hard. We formularize the problem as the experimental design of a linear regression model if we allow multiple measurements on a single node. By relaxing it to a convex optimization problem, we get the proportion of sample for each node given the budget of total sample size. Then, we use a probabilistic quantization to get the number of each node to be sampled. Moreover, we analyze how the sample size influences whether our object function is welldefined by perturbation analysis. Finally, we demonstrate the performance of the proposed approach through various numerical experiments.
\end{abstract}

Index Terms - Graph Signal, Sampling Theory, Convex Optimization, Experimental Design, Perturbation Analysis

\section{INTRODUCTION}

Graph signals (GS) are rather useful for describing signals and information in irregular domains, such as social, sensor and economic networks [1]. Graph signal processing (GSP) theory extends and generalizes the classic discrete signal processing theory to graphs by introducing graph Fourier transform [2]-[15], wavelet [16]-[20], etc. Two main approaches have been considered to construct a graph Fourier analysis framework: algebraic graph theory linked to the adjenacy matrix [2], [4]-[8] and spectral graph theory based on the graph Laplacian matrix [3], [8]-[15]. GSP theory have been developed to graph filter [13], [17], [20], community detection and clustering on graphs [11], [19], sampling and interpolation [3], [6], [7], [9], [10] and corresponding distributed algorithm [8], [12]. Recently, stationary processes to the graph domain has been investigated in [14], [15].

Sampling theory for GS deals with the problem of recovering a bandlimited signal from its samples on a subset of nodes of the graph. Bandlimited GS widely exist in most real-world networks due to the fact that the signals on adjacent nodes won't differ dramatically in reality. To formulate a sampling theory for bandlimited GS, the following questions arises: How to choose the best sampling set which can achieve the smallest reconstruction error? Generally, the signals can be bandlimited or approximate bandlimited and the samples can be noise-free or noisy as shown in Table. 1. In this paper, we focus on choosing the sampling set for bandlimited GS from noisy measurements.

Table 1. Literature based on different assumptions

\begin{tabular}{|c|c|c|}
\hline & Noise-free & Noisy \\
\hline Bandlimited & [6], [7] & [3], [7]-[10], [12] \\
\hline Approximate bandlimited & {$[6]$} & [2], [3] \\
\hline
\end{tabular}

In this work, we connect the design of sampling set for noisy bandlimited GS with experimental design problem, which is closely related to the optimal experimental design problem in [21], [22]. This connection with experimental design has also been noticed in [3], [8] for GS sampling but not fully investigated. The original form of experimental design problem with given measurement size is a combinatorial problem and NP-hard. There are two main kinds of solutions. One is heuristic algorithms, which is timeconsuming and can hardly produce an optimal solution, such as Fedorovs exchange algorithm [23] and Wynns algorithm [24]. The other is the convex relaxation proposed in [25, Sec. 7.5] which tries to obtain an optimal solution. It has been also claimed to give excellent results in many applications [26]. In this paper, we follow the latter.

In this work, we consider the case when any node is allowed to be sampled multiple times. From the view of experimental design, the optimal solution usually achieves when we allow multiple-time sampling. This is very feasible for many real-world networks such as sensor network and social network. We not only decide the nodes to be sampled but also get the proportion of sample for each node of a given sample size by relaxing the combinational problem to convex optimization. In order to get the number of each node to be sampled, we use probabilistic quantization [27] to unbiased quantify the solution of the relaxed problem to integers to get a suboptimal solution of the combinational problem. Different from the heuristic algorithm [3], [8] whose performance can not be evaluated, we take the quantization error as the perturbation and analyze how sample size influence the performance of our algorithm by perturbation analysis. Moreover, we find a lower bound of the sample size to ensure the object function of our algorithm well-defined, which can provide a reference to practical problems. Finally, the performance of our method is analyzed and shown to have smaller reconstruction error and is more robust against 
additive noise.

\section{SYSTEM MODEL}

Consider an $N$-vertex undirected connected graph $\mathcal{G}=$ $(\mathcal{V}, \mathcal{E}, \boldsymbol{W})$, where $\mathcal{V}$ is the vertex set, $\mathcal{E}$ is the edge set, and $\boldsymbol{W}$ is the weighted adjacency matrix. If there is an edge $e=(i, j)$ between vertices $i$ and $j$, then the entry $W_{i, j}$ represents the weight of the edge; otherwise $W_{i, j}=0$. A signal $f$ defined on the vertices of a graph can be represented as a vector $\boldsymbol{f} \in \mathbb{R}^{N}$, and its element $f_{i}$ represents the signal value at the $i$ th vertex in $\mathcal{V}$.

The graph Laplacian is defined as $\boldsymbol{L}=\boldsymbol{D}-\boldsymbol{W}$, where the degree matrix $\boldsymbol{D}=\operatorname{diag}(\mathbf{1} \boldsymbol{W})$. Since the Laplacian matrix is real symmetric, it has a complete eigenbasis and the spectral decomposition

$$
\boldsymbol{L}=\boldsymbol{V} \boldsymbol{\Lambda} \boldsymbol{V}^{T},
$$

where the eigenvectors $\left\{v_{k}\right\}_{0<k \leq N-1}$ of $\boldsymbol{L}$ form the columns of $\boldsymbol{V}$, and $\boldsymbol{\Lambda} \in \mathbb{C}^{N \times N}$ is a diagonal matrix of eigenvalues $0=\lambda_{0} \leq \lambda_{1} \leq \cdots \leq \lambda_{N-1}$ of $\boldsymbol{L}$. The graph Fourier transform corresponds to the basis expansion of a signal. The eigenvectors of the graph Laplacian are regarded as the Fourier basis and the eigenvalues are regarded as frequencies [5]. The expansion coefficients of a graph signal $f$ in terms of eigenvectors are defined as $\hat{f}$, so that a graph signal can be expressed as

$$
\boldsymbol{f}=\boldsymbol{V} \hat{\boldsymbol{f}} .
$$

A graph signal is called bandlimited when there exists a $K \in\{0,1, \cdots, N-1\}$ such that its graph Fourier transform $\hat{\boldsymbol{f}}$ satisfies $\hat{f}_{k}=0$ for all $k \geq K$ [7]. Let $\boldsymbol{f}$ be a signal with bandwidth $K$, then it satisfies

$$
\boldsymbol{f}=\boldsymbol{V}_{K} \hat{\boldsymbol{f}}_{K},
$$

where $\boldsymbol{V}_{K}$ denotes the first $K$ columns of $\boldsymbol{V}$ and $\hat{\boldsymbol{f}}_{K}$ denotes the first $K$ coefficients of $\hat{f}$.

Suppose that we sample $M$ measurements from the graph signal $\boldsymbol{f} \in \mathbb{C}^{N}$ to produce a sampled signal $\boldsymbol{f}_{\mathcal{S}} \in \mathbb{C}^{M}$, usually $M \leq N$, where $\mathcal{S}=\left(\mathcal{S}_{1}, \cdots, \mathcal{S}_{M}\right)$ denotes the sequence of sampled indices, and $\mathcal{S}_{i} \in\{1,2, \cdots, N\}$. The sampling operator $\Psi: \mathbb{C}^{N} \mapsto \mathbb{C}^{M}$ is defined as

$$
\Psi_{i, j}=\left\{\begin{array}{lc}
1, & j=\mathcal{S}_{i} \\
0, & \text { otherwise }
\end{array}\right.
$$

Let $\boldsymbol{w} \in \mathbb{C}^{M}$ be the i.i.d. noise with zero mean and unit variance introduced during sampling. Then the samples are given by $f_{\mathcal{S}}=\Psi f$, and the observation model is $\boldsymbol{y}_{\mathcal{S}}=\Psi \boldsymbol{f}+\boldsymbol{w}$. Considering (3), for bandlimited GS, the observation model can be expressed as $\boldsymbol{y}_{\mathcal{S}}=\Psi \boldsymbol{V}_{K} \hat{\boldsymbol{f}}_{K}+\boldsymbol{w}$. Let $V_{M K}=\Psi V_{K}$, then

$$
\boldsymbol{y}_{\mathcal{S}}=\boldsymbol{V}_{M K} \hat{\boldsymbol{f}}_{K}+\boldsymbol{w} .
$$

The best linear unbiased estimation [28] of $\hat{\boldsymbol{f}}_{K}$ from observed samples $\boldsymbol{y}_{\mathcal{S}}$ is,

$$
\hat{\boldsymbol{f}}_{K}^{\prime}=\boldsymbol{V}_{M K}^{\dagger} \boldsymbol{y}_{\mathcal{S}},
$$

where $\boldsymbol{V}_{M K}^{\dagger}=\left(\boldsymbol{V}_{M K}^{T} \boldsymbol{V}_{M K}\right)^{-1} \boldsymbol{V}_{M K}^{T}$ is the pseudo-inverse of $\boldsymbol{V}_{M K}$.

By (2), the estimation error of $f$ is

$$
\boldsymbol{e}=\boldsymbol{f}^{\prime}-\boldsymbol{f}=\boldsymbol{V}_{K} \boldsymbol{V}_{M K}^{\dagger} \boldsymbol{w} .
$$

The covariance matrix of estimation error is

$$
\boldsymbol{E}=\mathbb{E}\left[\boldsymbol{e} \boldsymbol{e}^{T}\right]=\boldsymbol{V}_{K}\left(\boldsymbol{V}_{M K}^{\mathrm{T}} \boldsymbol{V}_{M K}\right)^{-1} \boldsymbol{V}_{K}^{T} .
$$

Our main problem is to choice an optimal $\Psi$ that minimize the error covariance $\boldsymbol{E}$ in certain scalarization forms.

Several scalarizations of (8) have been proposed for the convenience of minimization as follow:

\section{$D$-optimal}

$$
f(\boldsymbol{E})=\log \operatorname{det}(\boldsymbol{E}),
$$

E-optimal

$$
f(\boldsymbol{E})=\|\boldsymbol{E}\|_{2},
$$

$A$-optimal

$$
f(\boldsymbol{E})=\operatorname{Tr}(\boldsymbol{E}) .
$$

It is obvious that $f(\boldsymbol{E})=f\left(\left(\boldsymbol{V}_{M K}^{\mathrm{T}} \boldsymbol{V}_{M K}\right)^{-1}\right)$ for all the three scalarizations above, since $V_{K}$ is orthogonal.

\section{ALGORITHM}

In this section, we consider the sampling set design problem by estimating $\boldsymbol{f}$ from measurements. Let $\boldsymbol{u}_{1}^{T}, \cdots, \boldsymbol{u}_{N}^{T}$ be the rows of $\boldsymbol{V}_{K}$. Recall from $\boldsymbol{V}_{M K}=\Psi \boldsymbol{V}_{K}$ that the the rows of $\boldsymbol{V}_{M K}$, which characterize the measurements, can be chosen among $N$ possible test vectors $\boldsymbol{u}_{1}^{T}, \cdots, \boldsymbol{u}_{N}^{T}$. Our goal of optimal experimental design is to make $f(\boldsymbol{E})$ as small as possible.

Let $m_{i}$ denotes the number of experiments for which $\boldsymbol{u}_{i}$ is chosen, and assume that the sample size is $M$, so we have

$$
m_{1}+\cdots+m_{N}=M .
$$

The scalarization of error covariance matrix can be expressed as

$$
f(\boldsymbol{E})=f\left(\left(\sum_{i=1}^{N} m_{i} \boldsymbol{u}_{i} \boldsymbol{u}_{i}^{T}\right)^{-1}\right) .
$$

It shows that the error covariance depends only on the numbers of each $\boldsymbol{u}_{i}$ is chosen. The basic experimental design problem is as follow,

$$
\begin{array}{cl}
\underset{m_{i}}{\operatorname{minimize}} & f(\boldsymbol{E}) \\
\text { subject to } & m_{i} \geq 0, \quad m_{1}+\cdots+m_{N}=M \\
& m_{i} \in \boldsymbol{Z} .
\end{array}
$$




\subsection{THE RELAXED PROBLEM}

The basic experimental design problem (14) is an intractable combinatorial problem. We relax the constraint that the $m_{i}$ are integers following Boyd's method [25, Sec. 7.5]. Let $p_{i}=m_{i} / M$, which indicates the proportion of experiment $i$, and relax the constraint that each $p_{i}$ is an integer multiple of $\frac{1}{M}$, we obtain the relaxed experimental design problem

$$
\begin{array}{ll}
\underset{p_{i}}{\operatorname{minimize}} & f(\boldsymbol{E})=f\left(\left(\sum_{i=1}^{N} p_{i} \boldsymbol{u}_{i} \boldsymbol{u}_{i}^{T}\right)^{-1}\right) \\
\text { subject to } & \boldsymbol{p} \succeq 0, \quad \mathbf{1}^{T} \boldsymbol{p}=1 .
\end{array}
$$

This is a convex optimization which can be solved by any optimization tool like interior-point methods [25]. In the rest of this paper, we consider only the relaxed experimental problem. The optimal value of the relaxed problem (15) provides a lower bound on the optimal value of the combinatorial one since the combinatorial problem has an addition constraint.

After solving (15), each entry of $p$ need to be quantified to an integer multiple of $\frac{1}{M}$. Different form Boyd's method [25, Sec. 7.5], we use probabilistic quantization [27] instead of the simple rounding to ensure an unbiased quantization in mean. The probabilistic quantization $Q: p_{i} \rightarrow Q\left(p_{i}\right)$ is defined as follow: $p_{i} \in[0,1]$ is equally divide into $M-1$ sub-intervals. The quantization points is defined as $\{1 / M, 2 / M, \ldots, 1\}$. Then, for $p_{i} \in[k / M,(k+1) / M]$, $k \in\{0,1, \ldots, M-1\}, Q\left(p_{i}\right)$ is a random variable defined by

$$
Q\left(p_{i}\right)= \begin{cases}\frac{k}{M} & \text { with probability }\left(\frac{k+1}{M}-p_{i}\right) M \\ \frac{k+1}{M} & \text { with probability }\left(p_{i}-\frac{k}{M}\right) M .\end{cases}
$$

Clearly we have $\left|p_{i}-Q\left(p_{i}\right)\right| \leq 1 /(2 M)$. So when $M$ is large enough, we have $\boldsymbol{p} \approx Q(\boldsymbol{p})$, which implies the error covariance matrix associated with $\boldsymbol{p}$ and $Q(\boldsymbol{p})$ are closed.

As a result, we can use $Q(\boldsymbol{p})$ to generate a suboptimal sampling set: let $m_{i}=M Q\left(\boldsymbol{p}_{i}\right)$ be the sample quota of the $i$ th node.

\subsection{PERTURBATION ANALYSIS OF QUANTIZA- TION}

By relaxing the experimental design problem, we do not need to solve the combinatorial problem directly. Meanwhile, the suboptimal solution brings a new problem of how to ensure the objective function of (15) invertible when we using $Q\left(p_{i}\right)$ to replace $p_{i}$, since the object function will be ill-condition if $\sum_{i=1}^{N} Q\left(p_{i}\right) \boldsymbol{u}_{i} \boldsymbol{u}_{i}^{T}$ is not invertible.

Let $\boldsymbol{A}=\sum_{i=1}^{N} p_{i} \boldsymbol{u}_{i} \boldsymbol{u}_{i}^{T}$ and $\hat{\boldsymbol{A}}=\sum_{i=1}^{N} Q\left(p_{i}\right) \boldsymbol{u}_{i} \boldsymbol{u}_{i}^{T}$, our goal is to ensure $\hat{\boldsymbol{A}}$, perturbed from $\boldsymbol{A}$, invertible. Let the perturbation error $\Delta p_{i}=Q\left(p_{i}\right)-p_{i}$, and the perturbation is given by

$$
\delta \boldsymbol{A}=\hat{\boldsymbol{A}}-\boldsymbol{A}=\sum_{i=1}^{N} \Delta p_{i} \boldsymbol{u}_{i} \boldsymbol{u}_{i}^{T}
$$

According to the [29, Th 2.1], known that $\boldsymbol{A}$ is nonsingular, the relative $l_{2}$-norm distance from $\boldsymbol{A}$ to the nearest singular matrix is

$$
\min \left\{\frac{\|(\delta \boldsymbol{A})\|_{2}}{\|\boldsymbol{A}\|_{2}}: \boldsymbol{A}+\delta \boldsymbol{A} \text { singular }\right\}=\frac{1}{\|\boldsymbol{A}\|_{2}\left\|\boldsymbol{A}^{-1}\right\|_{2}} .
$$

Therefore, to ensure $\hat{\boldsymbol{A}}$ invertible, the following condition needs to be satisfied,

$$
\|\delta \boldsymbol{A}\|_{2}<\frac{1}{\left\|\boldsymbol{A}^{-1}\right\|_{2}}=\sigma_{\min }(\boldsymbol{A}) .
$$

Recall from (17) that $\|\delta \boldsymbol{A}\|_{2}$ is related to $\Delta p_{i}$. Thus, $\Delta p_{i}$ affects the probability of $(19)$ being held.

Theorem 1. Suppose that $\Delta p_{1}, \ldots, \Delta p_{N}$ are independent, and each $Q\left(p_{i}\right)$ is obtained by the quantization given in (16). The probability of (19) is

$$
P\left(\|\delta \boldsymbol{A}\|_{2}<\sigma_{\min }(\boldsymbol{A})\right)>\prod_{i=1}^{N}\left(1-\frac{\operatorname{Var}\left[\Delta p_{i}\right]}{\left(\sigma_{\min }(\boldsymbol{A})\right)^{2}}\right) .
$$

Proof: Let $\Delta \boldsymbol{P}=\operatorname{diag}\left(\Delta p_{1}, \Delta p_{2}, \ldots, \Delta p_{N}\right)$, then

$$
\begin{aligned}
\|\delta \boldsymbol{A}\|_{2} & =\left\|\boldsymbol{V}_{k}^{T} \Delta \boldsymbol{P} \boldsymbol{V}_{k}\right\|_{2} \\
& \leq\left\|\boldsymbol{V}_{k}^{T}\right\|_{2}\|\Delta \boldsymbol{P}\|_{2}\left\|\boldsymbol{V}_{k}\right\|_{2}=\max \left|\Delta p_{i}\right| .
\end{aligned}
$$

Since $\Delta p_{1}, \ldots, \Delta p_{N}$ are independent, we can get the following inequation,

$$
P\left(\|\delta \boldsymbol{A}\|_{2}<\sigma_{\min }(\boldsymbol{A})\right) \geq \prod_{i=1}^{N} P\left(\left|\Delta p_{i}\right|<\sigma_{\min }(\boldsymbol{A})\right) .
$$

According to Chebycheff inequality, for any $p_{i} \in[k / M,(k+$ $1) / M]$, the following inequation holds,

$$
P\left(\left|\Delta p_{i}\right|<\sigma_{\min }(\boldsymbol{A})\right)>1-\frac{\operatorname{Var}\left[\Delta p_{i}\right]}{\left(\sigma_{\min }(\boldsymbol{A})\right)^{2}} .
$$

Thus proving our claim.

Assume that $M$ is large enough, then any $p_{i} \in$ $[k / M,(k+1) / M]$ is approximated to be a uniform distribution: $p_{i} \sim U[k / M,(k+1) / M]$. According to $[16)$, for every $p_{i} \in[k / M,(k+1) / M), \mathbb{E}\left[Q\left(p_{i}\right)\right]=p_{i}$, which means $Q\left(p_{i}\right)$ is an unbiased representation of $p_{i}$, so

$$
\operatorname{Var}\left[\Delta p_{i}\right]=\int_{-\frac{1}{2 M}}^{\frac{1}{2 M}} \Delta p_{i}{ }^{2} \mathrm{p}\left(\Delta p_{i}\right) \mathrm{d} \Delta p_{i} \approx \frac{5}{192 M^{3}} .
$$

We do not need the probability in $(20)$ to be 1 most of the time, it can be reduced to $\eta(0<\eta<1)$ according to actual needs. This leads to the following corollary.

Corollary 1. $\hat{\boldsymbol{A}}=\sum_{i=1}^{N} Q\left(p_{i}\right) \boldsymbol{u}_{i} \boldsymbol{u}_{i}^{T}$ is invertible with probability $\eta$ if the sample size $M$ satisfies:

$$
M \geq\left\lceil\left(\frac{5}{192(1-\sqrt[N]{\eta})\left(\sigma_{\min }(\boldsymbol{A})\right)^{2}}\right)^{\frac{1}{3}}\right\rceil,
$$

where $\lceil\cdot\rceil$ denotes the ceiling operation. 


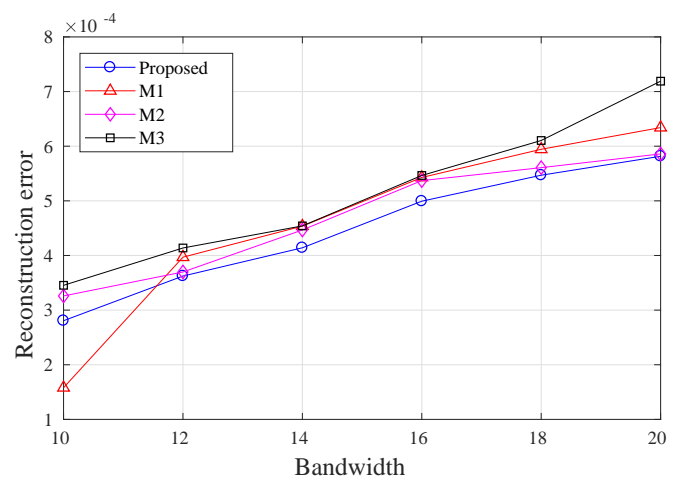

(a) Signal F1

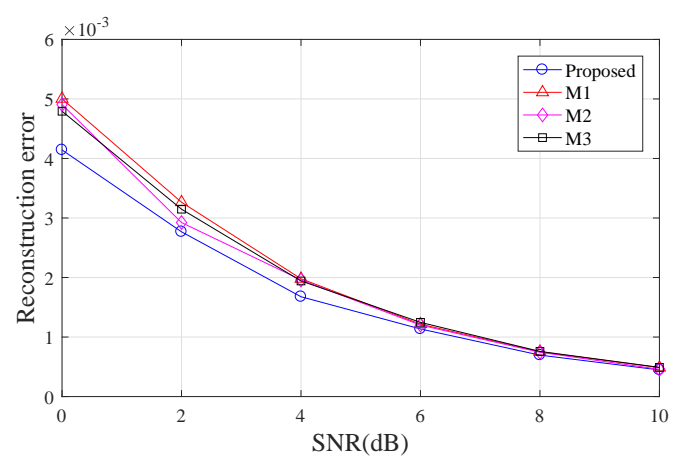

(b) Signal F2

Fig. 1. Reconstruction results of G1 for different signals.

\section{SIMULATION}

We now numerically evaluate the performance of the proposed work. The experiments compares the average reconstruction error $\|\boldsymbol{e}\|_{2}$ of different sample set selection algorithms versus different bandwidth of the true signal and different SNR of the samples. We compare our approach with the following methods: M1 [7] and M2 [3], which are both greedy algorithm, as well as M3 [26], which uses relaxed convex optimization to calculate the probability to sample each node and select $M$ nodes with largest probability.

We give some simulation results on the following simulated undirected graphs: Small world graph (G1) [30] of 1000 nodes rewiring with probability 0.1 . Random geometric graph (G2) [31] with 500 nodes placed randomly in the unit square and edges are placed between any nodes within 0.6. The edge weights are assigned via a Gaussian kernel.

For each of the above graphs, we consider the problem in the following scenarios: (F1) The bandwidth of the true signal varying from 10 to 20 and non-zero GFT coefficients are generated from $\mathcal{N}\left(1,0.5^{2}\right)$. The samples are noisy with additive i.i.d. Gaussian noise such that the SNR equals 10 dB. (F2) The true signal is exactly with the bandwidth of 15 and non-zero GFT coefficients are generated from $\mathcal{N}\left(1,0.5^{2}\right)$. The samples are noisy with additive $i . i . d$. Gaussian noise and the SNR varies from $0 \mathrm{~dB}$ to $10 \mathrm{~dB}$.

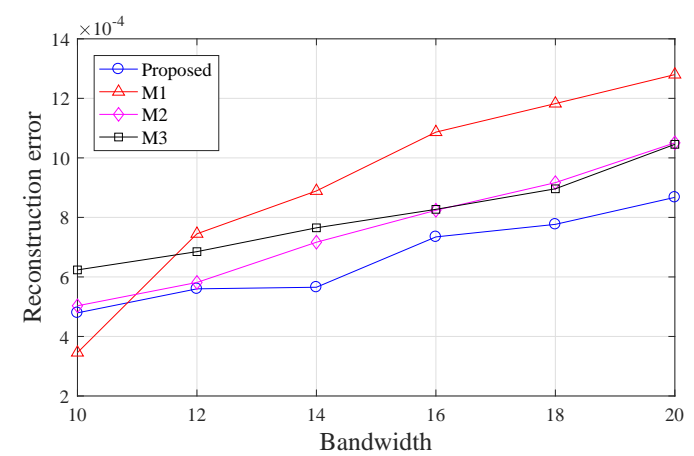

(a) Signal F1

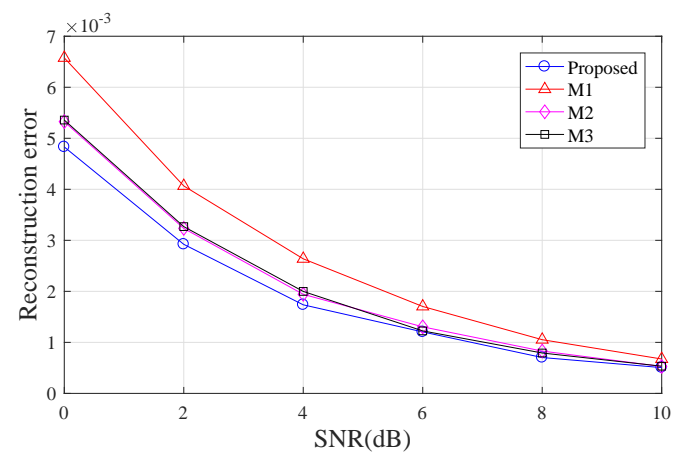

(b) Signal F2

Fig. 2. Reconstruction results of G2 for different signals.

We generate 200 signals from each of the two signal models on each of the graphs and set the sample size to 4 times of the bandwidth. The parameter $k$ of $\mathrm{M} 2$ that controls how closely the estimate the bandwidth of a signal is set to 8 in our simulation. For noisy signal model F1, our method has a better performance in reconstruction error than the others with the same sample size in most case as demonstrated in Fig. (1a) and Fig. (2a). This is because M1 and M2 are heuristic methods, which can not ensure a global optimal solution. M3 formularizes the problem as an experimental design problem like what we do but not allows multiple-time sampling on any node. So their solution is a suboptimal solution from the view of measurement, while we try to find the optimal one. The quantization step is a major factor that influences the performance of our method. The smaller sample size $M$ is, the larger the quantization step is. As a result, the corresponding reconstruction performance will be poorer. Our method also has a better performance for signal model F2, especially when the SNR of samples is low as demonstrated in Fig. (1b) and Fig. (2b). This also proves that our method is more robust against additive noise.

\section{CONCLUSION}

In this paper, an algorithm is proposed to obtain the best sampling set for bandlimited GS estimation for noisy samples. By the method of experimental design, we find out the sample quota for each node. Meanwhile, a loose lower 
bound of sample size is given to ensure the object function in our method is well-defined.

\section{Acknowledgment}

This work is supported by the NSF of China (Grant No. 61501124).

\section{REFERENCES}

[1] David I Shuman, Sunil K Narang, Pascal Frossard, Antonio Ortega, and Pierre Vandergheynst, "The emerging field of signal processing on graphs: Extending highdimensional data analysis to networks and other irregular domains," IEEE Signal Process. Mag., vol. 30, no. 3, pp. 83-98, 2013.

[2] Siheng Chen, Rohan Varma, Aarti Singh, and Jelena Kovačević, "Signal recovery on graphs: Fundamental limits of sampling strategies," IEEE Trans. Signal Inf. Process. Netw., vol. 2, no. 4, pp. 539-554, 2016.

[3] Aamir Anis, Akshay Gadde, and Antonio Ortega, "Efficient sampling set selection for bandlimited graph signals using graph spectral proxies," IEEE Trans. Signal Process., vol. 64, no. 14, pp. 3775-3789, 2016.

[4] Aliaksei Sandryhaila and José MF Moura, "Discrete signal processing on graphs," IEEE Trans. Signal Process., vol. 61, no. 7, pp. 1644-1656, 2013.

[5] Aliaksei Sandryhaila and José MF Moura, "Discrete signal processing on graphs: Frequency analysis," IEEE Trans. Signal Process., vol. 62, no. 12, pp. 30423054, 2014.

[6] S. K. Narang, A. Gadde, and A. Ortega, "Signal processing techniques for interpolation in graph structured data," in Proc. IEEE Int. Conf. Acoust., Speech, Signal Process. (ICASSP), 2013, pp. 5445-5449.

[7] Siheng Chen, Rohan Varma, Aliaksei Sandryhaila, and Jelena Kovačević, "Discrete signal processing on graphs: Sampling theory," IEEE Trans. Signal Process., vol. 63, no. 24, pp. 6510-6523, 2015.

[8] Antonio G Marques, Santiago Segarra, Geert Leus, and Alejandro Ribeiro, "Sampling of graph signals with successive local aggregations," IEEE Trans. Signal Process., vol. 64, no. 7, pp. 1832-1843, 2016.

[9] Gilles Puy, Nicolas Tremblay, Rémi Gribonval, and Pierre Vandergheynst, "Random sampling of bandlimited signals on graphs," Appl. Comput. Harmon. Anal., 2016.

[10] Mikhail Tsitsvero, Sergio Barbarossa, and Paolo Di Lorenzo, "Signals on graphs: Uncertainty principle and sampling," IEEE Trans. Signal Process., vol. 64, no. 18, pp. 4845-4860, 2016.

[11] Xiaowen Dong, Pascal Frossard, Pierre Vandergheynst, and Nikolai Nefedov, "Clustering on multi-layer graphs via subspace analysis on grassmann manifolds," IEEE Trans. Signal Process., vol. 62, no. 4, pp. 905-918, 2014.

[12] Xiaohan Wang, Pengfei Liu, and Yuantao Gu, "Localset-based graph signal reconstruction," IEEE Trans. Signal Process., vol. 63, no. 9, pp. 2432-2444, 2015.

[13] Xuesong Shi, Hui Feng, Muyuan Zhai, Tao Yang, and $\mathrm{Bo} \mathrm{Hu}$, "Infinite impulse response graph filters in wireless sensor networks," IEEE Signal Process. Lett., vol. 22, no. 8, pp. 1113-1117, 2015.

[14] Benjamin Girault, "Stationary graph signals using an isometric graph translation," in Proc. European Signal Processing Conf. (EUSIPCO), 2015, pp. 1516-1520. 
[15] Nathanaël Perraudin and Pierre Vandergheynst, "Stationary signal processing on graphs," IEEE Trans. Signal Process., vol. 65, no. 13, pp. 3462-3477, 2017.

[16] David K Hammond, Pierre Vandergheynst, and Rémi Gribonval, "Wavelets on graphs via spectral graph theory," Appl. Comput. Harmon. Anal., vol. 30, no. 2, pp. 129-150, 2011.

[17] Sunil K Narang and Antonio Ortega, "Compact support biorthogonal wavelet filterbanks for arbitrary undirected graphs," IEEE Trans. Signal Process., vol. 61, no. 19, pp. 4673-4685, 2013.

[18] David I Shuman, Christoph Wiesmeyr, Nicki Holighaus, and Pierre Vandergheynst, "Spectrum-adapted tight graph wavelet and vertex-frequency frames," IEEE Trans. Signal Process., vol. 63, no. 16, pp. 42234235, 2015.

[19] Nicolas Tremblay and Pierre Borgnat, "Graph wavelets for multiscale community mining," IEEE Trans. Signal Process., vol. 62, no. 20, pp. 5227-5239, 2014.

[20] Sunil K Narang and Antonio Ortega, "Perfect reconstruction two-channel wavelet filter banks for graph structured data," IEEE Trans. Signal Process., vol. 60, no. 6, pp. 2786-2799, 2012.

[21] Valerii Vadimovich Fedorov, Theory of optimal experiments, Elsevier, 1972.

[22] Friedrich Pukelsheim, Optimal design of experiments, SIAM, 2006.

[23] Alan J Miller and Nam-Ky Nguyen, "Algorithm as 295: A fedorov exchange algorithm for d-optimal design," Appl. Statist., vol. 43, no. 4, pp. 669-677, 1994.

[24] Henry P Wynn, "Results in the theory and construction of d-optimum experimental designs," J. Roy. Statist. Soc. Series B (Methodological), vol. 34, no. 2, pp. 133147, 1972.

[25] Stephen Boyd and Lieven Vandenberghe, Convex optimization, Cambridge university press, 2004.

[26] Siddharth Joshi and Stephen Boyd, "Sensor selection via convex optimization," IEEE Trans. Signal Process., vol. 57, no. 2, pp. 451-462, 2009.

[27] Tuncer C Aysal, Mark Coates, and Michael Rabbat, "Distributed average consensus using probabilistic quantization," in Proc. IEEE/SP Workshop on Statist. Signal Process. Workshop (SSP), 2007, pp. 640-644.

[28] Steven M Kay, Fundamentals of statistical signal processing, volume I: Estimation Theory, Prentice Hall, 1993.

[29] James W Demmel, Applied numerical linear algebra, SIAM, 1997.

[30] Duncan J Watts and Steven H Strogatz, "Collective dynamics of small-worldnetworks," nature, vol. 393, no. 6684, pp. 440-442, 1998.

[31] Nathanaël Perraudin, Johan Paratte, David Shuman, Lionel Martin, Vassilis Kalofolias, Pierre Vandergheynst, and David K Hammond, "Gspbox: A toolbox for signal processing on graphs," arXiv preprint arXiv:1408.5781, 2014. 